\title{
Insulator-to-Metal Transition Induced by Mid- IR Vibrational Excitation in a Magnetoresistive Manganite
}

\author{
Matteo Rini ${ }^{1}$, Jiro Itatani ${ }^{1,2}$, Yasuhide Tomioka ${ }^{3}$, Yoshinori Tokura ${ }^{3}$, Robert W. \\ Schoenlein $^{1}$, and Andrea Cavalleri ${ }^{4}$ \\ ${ }^{1}$ Lawrence Berkeley National Laboratory, 1 Cyclotron Road, MS 2-300, Berkeley, CA \\ 94720 USA \\ ${ }^{2}$ Non-equilibrium Dynamics Project, ERATO, Japan Science and Technology Agency, c/o \\ 1-1 Oho, Tsukua 305-0801, Japan \\ ${ }^{3}$ Correlated Electron Research Center, AIST Tsukuba Central 4, Tsukuba, Ibaraki, 305- \\ 8562 Japan \\ ${ }^{4}$ Department of Physics, Clarendon Laboratory, University of Oxford, Parks Road, Oxford \\ OX1 3PU, United Kingdom \\ E-mail: mrini@lbl.gov
}

\begin{abstract}
Selective vibrational excitation of insulating $\operatorname{Pr}_{0.7} \mathrm{Ca}_{0.3} \mathrm{MnO}_{3}$ at $17 \mu \mathrm{m}$ triggers a transition to a metallic state. A four order of magnitude drop of the sample resistivity and ultrafast, nanosecond-lived reflectivity changes are observed.
\end{abstract}

\section{Introduction}

In perovskite manganites the strong correlation between charge, spin, orbital and lattice degrees of freedom produces complex phase diagrams as well as extreme susceptibility to external stimuli, which can alter the balance between competing phases of the system. $\operatorname{Pr}_{0.7} \mathrm{Ca}_{0.3} \mathrm{MnO}_{3}$ has been a testing ground for a variety of phase-control concepts, whereby the transition from the charge-orbital ordered states to the ferromagnetic metallic state has been driven by external stimuli such as magnetic or electric field, high pressure and photoexcitation by x-rays or visible light [1]. Here we study the effect of selective excitation of an infrared active phonon mode, which is clearly shown to drive the solid toward the metallic phase. To our knowledge, this is the first example of an ultrafast insulator-metal transition driven in the electronic ground state.

A unique example among various manganites, $\operatorname{Pr}_{1-\mathrm{x}} \mathrm{Ca}_{\mathrm{x}} \mathrm{MnO}_{3}$ shows insulating behaviour over the whole composition $(\mathrm{x})$ range. This is a consequence of a more pronounced orthorhombic distortion, resulting in a small tolerance factor (see Fig. 1) [2]. The tolerance factor is a geometrical factor depending on the ( $\mathrm{Pr}, \mathrm{Ca})-\mathrm{O}$ and $\mathrm{Mn}-\mathrm{O}$ distances. It plays a key role in the physics of manganites, being related to the hopping probability of carriers between neighbouring $\mathrm{Mn}$ sites [2]. The infrared spectrum of $\operatorname{Pr}_{0.7} \mathrm{Ca}_{0.3} \mathrm{MnO}_{3}$ exhibits a typical pattern for manganites [3], with the highest frequency phonon assigned to a $\mathrm{Mn}-\mathrm{O}$ stretching vibration (Fig. 1). This mode modulates the geometrical parameters determining the tolerance factor and it is thus expected to have a strong coupling to the electronic system. 

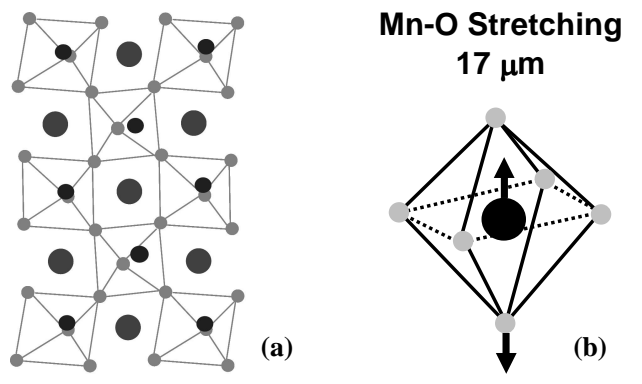

Fig 1. (a) Distorted orthorhombic structure of $\mathrm{Pr}_{0.7} \mathrm{Ca}_{0.3} \mathrm{MnO}_{3}$. (b) Atomic displacements within the $\mathrm{MnO}_{6}$ octahedra associated with the $17 \mu \mathrm{m}\left(580 \mathrm{~cm}^{-1}\right)$ phonon mode which modulates the $\mathrm{Mn}-\mathrm{O}$ distance and hence the tolerance factor.

\section{Electrical Measurements}

Changes in the sample conductivity were monitored by directly measuring the sample resistance following mid-infrared excitation at $17 \mu \mathrm{m}(200 \mathrm{fs}, 1 \mu \mathrm{J})$. The laser spot fully covered the space between two gold electrodes deposited on the sample surface. The sample was biased at a DC voltage of $27 \mathrm{~V}$ and cooled to 40 $\mathrm{K}$ (charge-ordered, anti-ferromagnetic insulator phase). Mid-infrared excitation induces a 50-fold current increase, corresponding to a resistance drop from 700 $\mathrm{M} \Omega$ to $15 \mathrm{M} \Omega$. The sample resistivity drops by more than four orders of magnitude, from the $40 \mathrm{~K}$ value of $310^{7} \Omega \cdot \mathrm{cm}$ to about $10^{3} \Omega \cdot($ see Fig. 2). The effect vanishes when the infrared pump wavelength is tuned outside the phonon resonance at $17 \mu \mathrm{m}$, including radiation at $8.5 \mu \mathrm{m}$ with twice as much energy/photon. The temperature jump due to laser excitation $(<2 \mathrm{~K})$ can be ruled out as origin of such a dramatic resistivity drop.

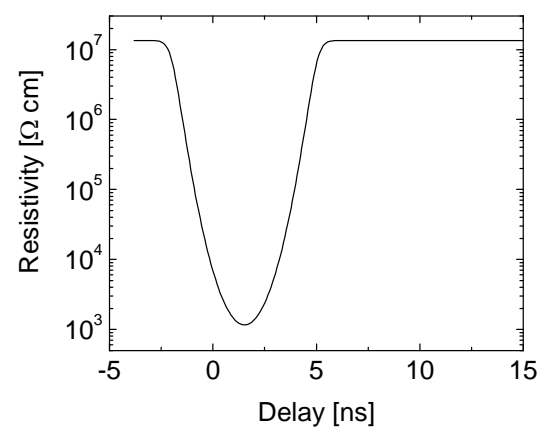

Fig 2. Resistivity change after excitation by $200 \mathrm{fs}, 17 \mu \mathrm{m}$ pulses. The dynamics of the relaxation process cannot be resolved with the 4 nsinstrumental response.

\section{Pump-probe Experiments}

The ultrafast dynamics of the photoinduced phase transition in $\operatorname{Pr}_{0.7} \mathrm{Ca}_{0.3} \mathrm{MnO}_{3}$ have been previously studied by means of femtosecond reflection spectroscopy [4]. The formation of the metallic phase following electronic excitation results in ultrafast $(<230 \mathrm{fs})$, long lived reflectivity changes in the near IR. Here, we show that vibrational excitation has comparable effects on the dynamics of the 
reflectivity changes. The sample was excited by $17 \mu \mathrm{m}$ pulses (fluence: $1 \mathrm{~mJ} / \mathrm{cm}^{2}$ ) and the reflectivity was probed by delayed $800 \mathrm{~nm}$ pulses. Fig. 3(a) shows the relative change of reflectivity $(\Delta R / R)$ as a function of pulse delay, evidencing an ultrafast reflectivity drop which persists up to the nanosecond timescale. Identical dynamics are observed following electronic excitation. Fig. 3(b) compares the spectrum of the reflectivity changes measured $1 \mathrm{ps}$ after vibrational excitation with the absorption spectrum around the phonon peak at $17 \mu \mathrm{m}$, showing that reflectivity changes depend on resonant infrared excitation of $\mathrm{Mn}-\mathrm{O}$ modes.
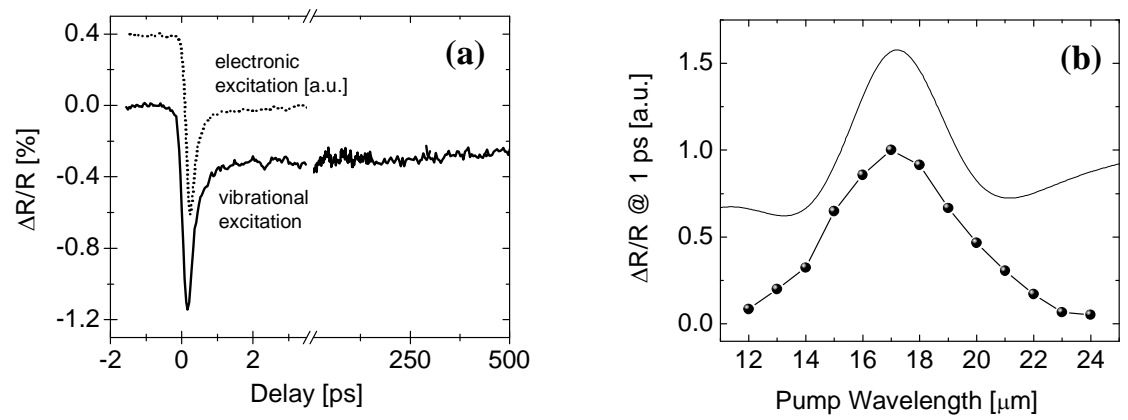

Fig.3. (a) Relative change of reflectivity at $800 \mathrm{~nm}(\Delta \mathrm{R} / \mathrm{R})$ as a function of pulse delay following electronic excitation (dotted line, normalized) and vibrational excitation at 17 $\mu \mathrm{m}$ (solid line). (b) $\Delta \mathrm{R} / \mathrm{R}$ measured $1 \mathrm{ps}$ after excitation (circles) and absorption spectrum around the $17 \mu \mathrm{m}$ phonon resonance (solid line). In order to compare the two curves, the phonon spectrum was convoluted with the spectrum of the broad bandwidth pump pulses.

\section{Conclusions}

The excitation of a specific phonon mode has been demonstrated as a tool to drive the solid in the electronic ground state toward a competing phase of the system. Ultrafast vibrational control of the collective phase of correlated electron systems opens a new window on the interaction between lattice structure and electronic or magnetic properties. This approach may extend well beyond the case of CMR manganites, providing new insight into the behavior of complex matter, including the controversial nature of High- $\mathrm{T}_{\mathrm{C}}$ superconductivity.

Acknowledgements. This work was supported by the U.S. Department of Energy under Contract No. DE-AC02-05CH11231.

\section{References}

1 Y. Tokura, Rep. Prog. Phys. 69, 797-851 (2006).

2 E. Dagotto, Nanoscale phase separation and colossal magnetoresistance (Springer-Verlag Berlin Heidelberg 2003).

3 Y. Okimoto et al., Phys. Rev. B 59, 7401-7408 (1999).

4 M. Fiebig et al., Appl. Phys. B, 71, 211-215 (2000). 